\title{
Nanoscopic Visualization of Cross-Linking Density in Polymer Networks with Diarylethene Photoswitches
}

\author{
Eric Siemes ${ }^{+}$, Oleksii Nevskyi $i^{+}$Dmytro Sysoiev, Sarah K. Turnhoff, Alex Oppermann, \\ Thomas Huhn, Walter Richtering, and Dominik Wöll*
}

\begin{abstract}
The in situ nanoscopic imaging of soft matter polymer structures is of importance to gain knowledge of the relationship between structure, properties, and functionality on the nanoscopic scale. Cross-linking of polymer chains effects the viscoelastic properties of gels. The correlation of mechanical properties with the distribution and amount of crosslinkers is relevant for applications and for a detailed understanding of polymers on the molecular scale. We introduce a super-resolution fluorescence-microscopy-based method for visualizing and quantifying cross-linker points in polymer systems. A novel diarylethene-based photoswitch with a highly fluorescent closed and a non-fluorescent open form is used as a photoswitchable cross-linker in a polymer network. As an example for its capability to nanoscopically visualize crosslinking, we investigate pNIPAM microgels as a system known with variations in internal cross-linking density.
\end{abstract}

$S_{\mathrm{u}}$ uper-resolution fluorescence microscopy methods allow in situ visualization and investigation of various biological structures at the nanoscale level. ${ }^{[1-7]}$ In recent years, an increasing number of soft matter systems and materials science questions can also be addressed, since the commonly employed methods for biological systems could be adapted for materials science. ${ }^{\left[{ }^{8,9]}\right.}$ Super-resolution fluorescence microscopy has the potential to give new insights that complement those obtained by well-established and recognized techniques in materials science, such as scanning probe microscopy techniques ${ }^{[10]}$ and modern electron microscopy methods. ${ }^{[11]}$ The scanning probe techniques give access to the nanometer range and determine softness and surface properties, such as topology, and the modern electron microscopy techniques can yield structural information even in the subnanometer range, provided there is sufficient electron density contrast. ${ }^{[12,13]}$ However, scanning probe and electron microscopy methods have the drawback, that they are invasive or need high-vacuum conditions. Additionally,

[*] E. Siemes, ${ }^{[+]}$O. Nevskyi, ${ }^{[+]}$S. K. Turnhoff, A. Oppermann, Prof. Dr. W. Richtering, Prof. Dr. D. Wöll

Institute for Physical Chemistry

RWTH Aachen University

Landoltweg 2, 52074 Aachen (Germany)

E-mail:woell@pc.rwth-aachen.de

Dr. D. Sysoiev, Dr. T. Huhn

Department of Chemistry, University of Konstanz

Universitätsstrasse 10, 78464 Konstanz (Germany)

$\left.{ }^{+}\right]$These authors contributed equally to this work. many polymer systems have poor electron contrast. The advantages of super-resolution fluorescence imaging techniques are that they are non-invasive and allow for real-time imaging of structures. ${ }^{[14,15]}$ For addressing many relevant questions in materials science, however, further developments concerning sample preparation, measurement conditions, and designing switchable dyes with suitable photophysical and chemical properties, and especially selective labeling capability of functionalities or compartments, are necessary. Direct visualization of the position of single crosslinks and their distribution in polymer networks is a dream of polymer physicists and chemists. It is known that in many polymer networks and hydrogels such cross-links exhibit heterogeneities on the nanoscale ${ }^{[16]}$ which are often already introduced by the polymerization conditions. ${ }^{[17]}$ The high complexity of such cross-linked soft matter systems prevents their quantitative structural analysis by common spectroscopic techniques. ${ }^{[18]}$

Polymer networks that are extensively swollen with water are the basis of hydrogels, ${ }^{[19]}$ both as macroscopic sizes and in their particle form as microgels in a size range from nanometers to several micrometers. ${ }^{[20]}$ In the swollen state, the volume of such a microgel is in many cases filled by less than $10 \%$ polymer. ${ }^{[21]}$ Choosing appropriate monomers, such as $\mathrm{N}$ isopropyl acrylamide (NIPAM), these microgels can be made stimuli-responsive and possess potential for a wide variety of applications, for example, as smart surface coatings and membranes, ${ }^{[22,23]}$ controlled release of pharmaceuticals or guest-molecules ${ }^{[24,25]}$ model systems for colloidal chemistry, ${ }^{[26]}$ (bio)catalysis, ${ }^{[27,28]}$ optical applications ${ }^{[29,30]}$ and emulsion stabilization. ${ }^{[31,32]}$ The physical properties of microgels are mainly governed by the cross-linker content and distribution. ${ }^{[33]}$ Scattering experiments indicate a complex morphology, often described by a so-called "fuzzy-sphere" model with dangling chains, ${ }^{[21]}$ for the internal structure of such a microgel, which is governed by polymerization kinetics. ${ }^{[34]}$ External stimuli, such as change in temperature, ionic strength, $\mathrm{pH}$, pressure, external magnetic and electric fields, or light provide the opportunity to reversibly change the physicochemical properties. However, this responsive behavior is also to a large extent governed by the network structure. Thus, a direct visualization and quantification of the cross-linker positions is a key point to understand complex microgel architectures and characterize their properties. ${ }^{[35]}$ It has been shown that super-resolution fluorescence microscopy is highly suitable for investigations of microgels with covalently bound labels ${ }^{[36-38]}$ or freely diffusing dye molecules penetrating the microgel network ${ }^{[39]}$ However, in all of these cases, switching buffers were essential to obtain the fluorescence blinking 
conditions required for localization-based super-resolution methods. Such buffers can affect the swelling especially in the case of microgels that contain ionic and $\mathrm{pH}$-sensitive moieties.

Herein, we introduced a photochromic diarylethene photoswitch as cross-linker into a microgel for super-resolution imaging. Not only has this dye never been used to address polymer networks and gels before, but we also show for the first time that cross-linker densities and distributions can be visualized with unprecedented precision using photoactivation localization microscopy (PALM) as a nanoscopy method. We demonstrate the high potential of this approach for imaging of the heterogeneous cross-linker distribution in pNIPAM-based microgels as one of the most prominent microgels and, in general, polymer network systems.

Diarylethenes, apart from their high potential in data storage, chemical sensing ${ }^{[40,41]}$ and their possibility to induce switching in other chromophores, ${ }^{[42]}$ have recently found application as a universal tool for super-resolution imaging. ${ }^{[4-45]}$ They possess spectrally well-separated on- and offstates, high absorption coefficients, high fluorescence quantum yield of the on-state, high photostability, a low quantum yield for the off-switching, and a reasonable quantum yield for on-switching.

We synthesized photoswitches 1 oc and 2 oc (Figure 1; $\mathbf{o}=$ open, $\mathbf{c}=$ closed) by a Suzuki-Miyaura coupling reaction using the 6,6'-diiodo derivative of 1,2-bis(2-ethyl-1,1-dioxidobenzothiophene-3-yl)perfluorocyclopentene and corresponding boronic acids according to the procedure previously described by Irie and co-workers (for more details see the Supporting Information). ${ }^{[46]}$ The absorption and fluorescence spectra of the corresponding closed forms $\mathbf{1 c}$ and 2c are presented in Figure 1. The bathochromic shift of the absorp-

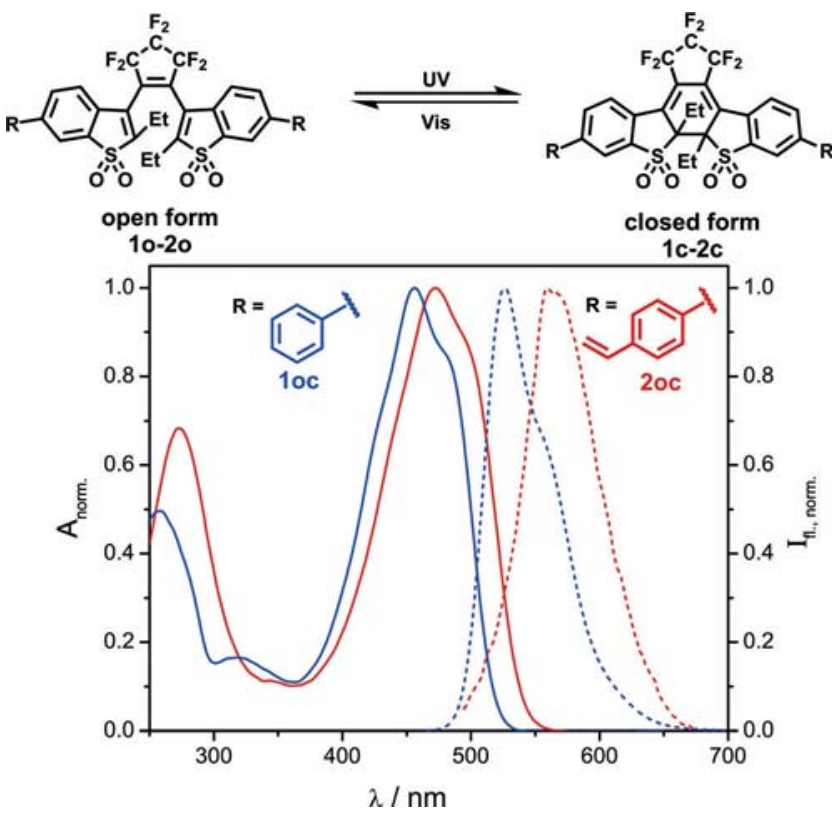

Figure 1. Chemical structures of the open and the closed form of photoswitches $10 c$ and 2 oc; absorption spectra of the closed forms $1 \mathrm{c}$ (blue solid line) and $2 \mathrm{c}$ (red solid line), and the corresponding fluorescence spectra of $1 \mathrm{c}$ (blue dashed line) and $2 \mathrm{c}$ (red dashed line). All spectra were recorded in 1,4-dioxane. tion band of the closed form $\mathbf{2} \mathbf{c}$ in comparison to $\mathbf{1 c}$ is due to an increase of the $\pi$-system length. Photoswitch 2 oc has two styrene units on both sides which allows for its incorporation as cross-linker in radical polymerizations. The diarylethene chromophore has been shown to be extremely stable under radical polymerization conditions. ${ }^{[47]}$ After the polymerization reaction, photoswitch $\mathbf{2}$ oc behaves similar to the phenyl derivative 1 oc owing to the negligible influence of the alkyl-substituents in the para-position of the phenyl groups. The photophysical properties of photoswitch $\mathbf{1 o c}$ are illustrated in Table S1 of the Supporting Information. The open and closed forms can be interconverted by irradiation with suitable wavelengths (for more details see Figure S1). The irradiation with UV-light mainly results in the formation of the closed form, while the irradiation with the visible light recovers the open form. The low off-switching (cycloreversion) quantum yield of the benzothiophene-1,1-dioxide-based diarylethene photoswitches is essential for PALM imaging. It allows for the collection of a high number of photons necessary for an accurate localization of the position ${ }^{[48]}$ of single photoswitches before they switch back to the dark state. 2 oc can be imaged with only one excitation wavelength as also shown for $\mathbf{1 0 c}$ by Irie and co-workers. ${ }^{[4]}$ This is of great practical advantage in various super-resolution fluorescence microscopy applications.

Imaging of cross-links with our novel diarylethene derivative $\mathbf{2}$ oc was performed on microgels, as they are highly relevant swollen polymer networks with a heterogeneous crosslinking density as determined by scattering techniques. ${ }^{[21]}$ pNIPAM-based microgels were synthesized by free radical precipitation polymerization with $N, N^{\prime}$-methylenebis(acrylamide) (BIS) and $\mathbf{2}$ oc as cross-linkers as shown in Figure 2. The amount of our DAE cross-linker 2 oc with respect to BIS was $4 \mathrm{~mol} \%$ in order not to modify the properties of the microgels significantly from the well-known and well-characterized microgels cross-linked with BIS. After synthesis, the microgels were purified via dialysis and centrifugation in order to minimize the amount of free dye to a non-significant amount. The detailed procedure can be found in the Supporting Information.

Super-resolved PALM imaging of the microgels containing 2 oc cross-linkers was performed on a widefield fluorescence microscope, the details of which can be found in the Supporting Information. 3D localization was obtained by astigmatism. ${ }^{[50]}$ For this purpose a cylindrical lens with $1000 \mathrm{~mm}$ focal length was placed at an appropriate position in the emission path, resulting in $z$-position dependent elongation of the single emitter point spread function along

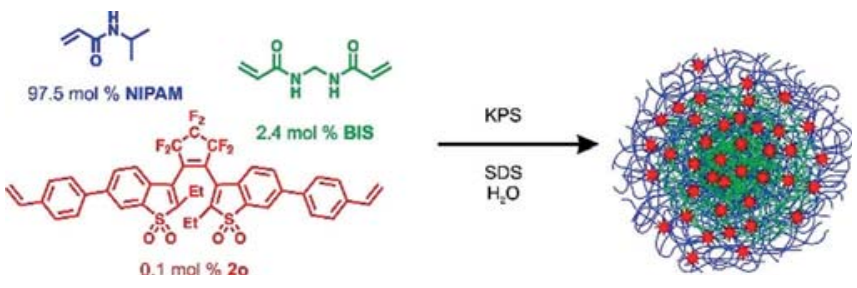

Figure 2. Synthesis of the pNIPAM-based microgels under investigation. $\mathrm{KPS}=$ potassium persulfate, $\mathrm{SDS}=$ sodium dodecyl sulfate. 
two axes. The excitation wavelength and power was set to $488 \mathrm{~nm}$ and approximately $6.25 \mathrm{~kW} \mathrm{~cm}^{-2}$, respectively. These conditions directly resulted in appropriate blinking conditions for PALM. Note that, in contrast to typically direct stochastic optical reconstruction microscopy (dSTORM) measurements, ${ }^{[51]}$ no additives to induce blinking were required. Movies of the blinking events with $10 \mathrm{~ms}$ integration time were recorded on an EMCCD camera (iXon Ultra 897) and analyzed with ThunderSTORM software. ${ }^{[52]}$ Superposition of single 3D localizations leads to a super-resolved image of the cross-links in microgels as shown in Figure 3. Every dot in Figure 3 corresponds to a single localization event. As an
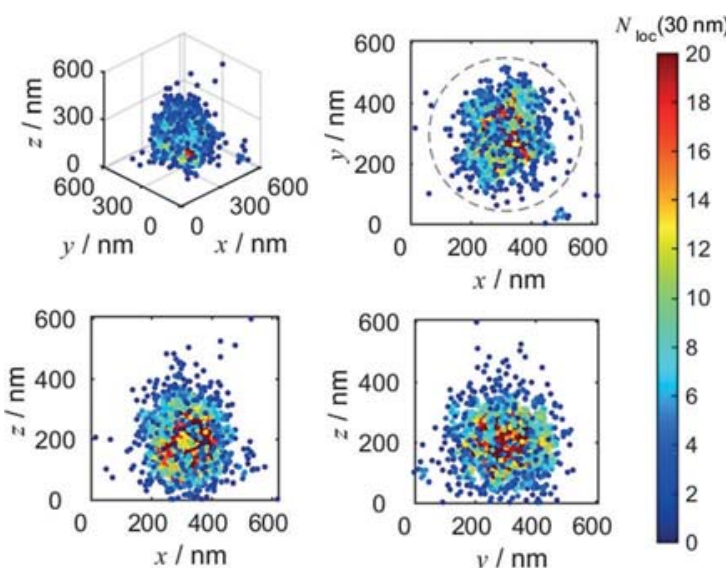

Figure 3. 3D distributions of the cross-linker positions of an individual microgel in its hydrated, swollen state and the corresponding 2D projections to three different planes. Each point represents a localization of a diarylethene cross-linker. The color scale indicates the number of localizations within a radius of $30 \mathrm{~nm}$, that is, the crosslinker density. The dashed gray circle illustrates the hydrodynamic radius $r_{\mathrm{h}}$ as determined by dynamic light scattering (see Figure S2). A 3D animation can be found in the Supporting Information.

additional way to illustrate the cross-linker density, the dots were color-coded according to the number of neighboring positions within a radius of $30 \mathrm{~nm}$. The resulting reconstructed images of the microgels exhibit a dense, highly crosslinked center and less cross-linked outer boundaries. In the center, a high amount of localizations is surrounded by 20 or more localizations within a $30 \mathrm{~nm}$ region, whereas single localizations can be found in the outer periphery of the microgel.

Beyond a visualization of the cross-linkers and their heterogeneous appearance, a thorough analysis of their distributions is of high theoretical as well as practical importance. In particular, for pNIPAM microgels, it has been found by different scattering methods that the polymer density decreases from the center towards the periphery. ${ }^{[21]}$ Additional indication by super-resolved dSTORM imaging with freely diffusing dyes was recently published by Bergmann et al. ${ }^{[39]}$ In contrast, we directly observed the positions of cross-links, and can correlate their appearance to the local microgel density. The density was determined by static light scattering (SLS). As shown in the upper graph of Figure 4, the static light scattering data can be fitted with the fuzzy sphere
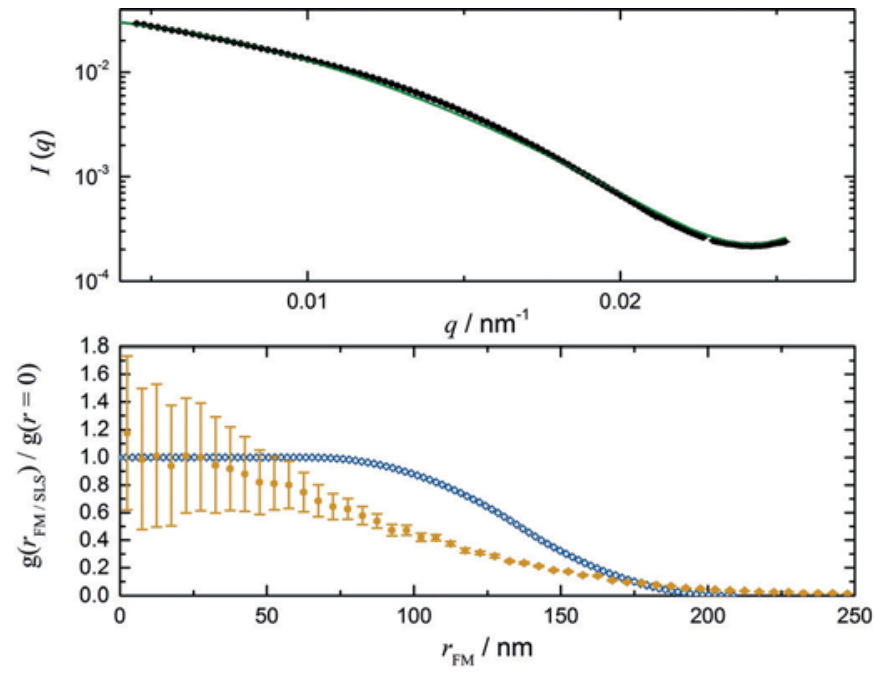

Figure 4. Upper graph: Static light scattering data of the p(NIPAM-coBIS-co-DAE)-microgel (black circles). The data were fitted with a fuzzy sphere model (green line, for more details see Supporting Information). Lower graph: Radial distribution of polymer density as obtained by the fit of the SLS-data (open blue diamonds) and radial distribution of DAE-cross-linkers obtained from PALM 3D-images (full orange circles), averaged over 20 microgels. Error bars indicate the standard deviation of the density obtained from single microgel images. All radial distributions were normalized by their value in the center.

model by Stieger et al. ${ }^{[21]}$ (for details of the parameter see the Supporting Information). In the lower graph of Figure 4 the resulting polymer density is shown along with the cross-linker density obtained by super-resolution microscopy. The radial distributions of the polymer density and cross-linker $\mathbf{2}$ are significantly different. In contrast to the polymer density, which can be well described as a fuzzy sphere, the radial density of cross-linker 2 levels off more rapidly and, within 30 to $150 \mathrm{~nm}$, in an almost linear way. The highest density of cross-linker $\mathbf{2}$ can be found in the microgel center where also the polymer density is at its maximum. Proceeding out of the center, the polymer density remains still rather constant until about $80 \mathrm{~nm}$ distance from the microgel center, whereas the relative density of cross-linker $\mathbf{2}$ already dropped off to around $50 \%$ at this distance. In the outer microgel regions, that is, the fuzzy region with dangling chains, both polymer and cross-linker $\mathbf{2}$ density are relatively low. Such a detailed comparison of the differences between polymer density and cross-linker density has never been performed before since it was not technically possible to disentangle both values, but our current approach allows this to be done. Note that any modification of a cross-linker can have significant influence on polymerization kinetics and, therefore, the way it is distributed. ${ }^{[53]}$ This is true for fluorescent cross-linkers introduced during polymerization or subsequent chemical reaction to a cross-linking group. Typical cross-linkers, such as BIS, however, do not allow for super-resolved imaging neither in fluorescence nor in electron microscopy. As a consequence, our approach is the only possibility to visualize single crosslinks, and future work will concentrate on combining the fluorescent cross-linker with typical ones in order to determine how polymerization conditions and the properties of the 
cross-linkers determine their distribution in the final polymer networks.

In summary, we developed a diarylethene photoswitch with two styrene groups which can act as cross-linker. This allows for a visualization and analysis of the cross-linker distribution with localization-based super-resolution fluorescence microscopy down to the nanoscale without the need for any additives that affect $\mathrm{pH}$ and ionic strength. We demonstrated the power of this approach with microgels which are known to exhibit inhomogeneous polymer density, but for which the cross-linker density has never been experimentally analyzed. It was shown that both polymer and cross-linker density decreases when proceeding from the center of a microgel to its periphery. This decrease shows a surprisingly different dependency, that is, the density of cross-linker $\mathbf{2}$ does not automatically allow for a deduction of polymer density and vice versa. How different polymerization rates of the crosslinker can account for this behavior is currently under investigation. However, conclusively, the method presented herein has high potential to set a new benchmark in the study of polymer networks and their heterogeneity.

\section{Acknowledgements}

We thank the Deutsche Forschungsgemeinschaft (DFG) for support within the projects C5 and A3 of the SFB 985 "Functional Microgels and Microgel Systems" and the RWTH profile area MSE (Molecular Science and Engineering) for supporting the competence center FLAMENCO (high resolution FLuorescence microscopy Applied in Molecular Science and ENgineering COmpetence center).

\section{Conflict of interest}

The authors declare no conflict of interest.

Keywords: cross-linker - microgels · photoswitch · soft matter . super-resolution fluorescence microscopy

[1] S. W. Hell, S. J. Sahl, M. Bates, X. Zhuang, R. Heintzmann, M. J. Booth, J. Bewersdorf, G. Shtengel, H. Hess, P. Tinnefeld, A. Honigmann, S. Jakobs, I. Testa, L. Cognet, B. Lounis, H. Ewers, S. J. Davis, C. Eggeling, D. Klenerman, K. I. Willig, G. Vicidomini, M. Castello, A. Diaspro, T. Cordes, J. Phys. D 2015, 48, 443001.

[2] E. Betzig, G. H. Patterson, R. Sougrat, O. W. Lindwasser, S. Olenych, J. S. Bonifacino, M. W. Davidson, J. LippincottSchwartz, H. F. Hess, Science 2006, 313, 1642-1645.

[3] W. R. Legant, L. Shao, J. B. Grimm, T. A. Brown, D. E. Milkie, B. B. Avants, L. D. Lavis, E. Betzig, Nat. Methods 2016, 13, 359365.

[4] M. Heilemann, S. van de Linde, M. Schüttpelz, R. Kasper, B. Seefeldt, A. Mukherjee, P. Tinnefeld, M. Sauer, Angew. Chem. Int. Ed. 2008, 47, 6172-6176; Angew. Chem. 2008, 120, $6266-$ 6271.

[5] S. W. Hell, Science 2007, 316, 1153-1158.
[6] M. J. Rust, M. Bates, X. Zhuang, Nat. Methods 2006, 3, 793-796.

[7] M. Heilemann, S. van de Linde, A. Mukherjee, M. Sauer, Angew. Chem. Int. Ed. 2009, 48, 6903-6908; Angew. Chem. 2009, 121, $7036-7041$.

[8] D. Wöll, C. Flors, Small Methods 2017, 1, 1700191.

[9] A. Aloi, I. K. Voets, Curr. Opin. Colloid Interface Sci. 2018, 34 , $59-73$.

[10] E. T. Herruzo, A. P. Perrino, R. Garcia, Nat. Commun. 2014, 5 , 3126.

[11] L. E. Franken, E. J. Boekema, M. C. A. Stuart, $A d v$. Sci. 2017, 4 , 1600476.

[12] M. T. Proetto, A. M. Rush, M.-P. Chien, P. Abellan Baeza, J. P. Patterson, M. P. Thompson, N. H. Olson, C. E. Moore, A. L. Rheingold, C. Andolina, J. Millstone, S. B. Howell, N. D. Browning, J. E. Evans, N. C. Gianneschi, J. Am. Chem. Soc. 2014, 136, $1162-1165$.

[13] K. Geisel, L. Isa, W. Richtering, Angew. Chem. Int. Ed. 2014, 53, 4905-4909; Angew. Chem. 2014, 126, 5005-5009.

[14] S. Onogi, H. Shigemitsu, T. Yoshii, T. Tanida, M. Ikeda, R. Kubota, I. Hamachi, Nat. Chem. 2016, 8, $743-752$.

[15] H. Deschout, T. Lukes, A. Sharipov, D. Szlag, L. Feletti, W. Vandenberg, P. Dedecker, J. Hofkens, M. Leutenegger, T. Lasser, A. Radenovic, Nat. Commun. 2016, 7, 13693.

[16] F. Di Lorenzo, S. Seiffert, Polym. Chem. 2015, 6, 5515-5528.

[17] D. Wöll, H. Uji-i, T. Schnitzler, J. I. Hotta, P. Dedecker, A. Herrmann, F. C. De Schryver, K. Müllen, J. Hofkens, Angew. Chem. Int. Ed. 2008, 47, 783-787; Angew. Chem. 2008, 120, 795 799.

[18] D. Estupiñán, C. Barner-Kowollik, L. Barner, Angew. Chem. Int. Ed. 2018, 57, 5925-5929; Angew. Chem. 2018, 130, 6028-6033.

[19] X. Du, J. Zhou, J. Shi, B. Xu, Chem. Rev. 2015, 115, 1316513307.

[20] L. A. Lyon, A. Fernandez-Nieves, Annu. Rev. Phys. Chem. 2012, 63, 25-43.

[21] M. Stieger, W. Richtering, J. S. Pedersen, P. Lindner, J. Chem. Phys. 2004, 120, 6197-6206.

[22] M. Kather, M. Skischus, P. Kandt, A. Pich, G. Conrads, S. Neuss, Angew. Chem. Int. Ed. 2017, 56, 2497-2502; Angew. Chem. 2017, 129, $2537-2543$.

[23] D. Menne, F. Pitsch, J. E. Wong, A. Pich, M. Wessling, Angew. Chem. Int. Ed. 2014, 53, 5706-5710; Angew. Chem. 2014, 126, 5814-5818

[24] R. A. Meurer, S. Kemper, S. Knopp, T. Eichert, F. Jakob, H. E. Goldbach, U. Schwaneberg, A. Pich, Angew. Chem. Int. Ed. 2017, 56, 7380-7386; Angew. Chem. 2017, 129, 7486-7492.

[25] L. Nuhn, M. Hirsch, B. Krieg, K. Koynov, K. Fischer, M. Schmidt, M. Helm, R. Zentel, ACS Nano 2012, 6, 2198-2214.

[26] K. Han, D. Go, T. Tigges, K. Rahimi, A. J. C. Kuehne, A. Walther, Angew. Chem. Int. Ed. 2017, 56, 2176-2182; Angew. Chem. 2017, 129, 2208-2214.

[27] S. Wiese, A. C. Spiess, W. Richtering, Angew. Chem. Int. Ed. 2013, 52, 576-579; Angew. Chem. 2013, 125, 604-607.

[28] S. Wu, J. Dzubiella, J. Kaiser, M. Drechsler, X. Guo, M. Ballauff, Y. Lu, Angew. Chem. Int. Ed. 2012, 51, 2229-2233; Angew. Chem. 2012, 124, 2272-2276.

[29] J. D. Debord, S. Eustis, S. Byul Debord, M. T. Lofye, L. A. Lyon, Adv. Mater. 2002, 14, 658-662.

[30] M. Chen, L. Zhou, Y. Guan, Y. Zhang, Angew. Chem. Int. Ed. 2013, 52, 9961-9965; Angew. Chem. 2013, 125, 10145-10149.

[31] H. Monteillet, M. Workamp, X. Li, B. Schuur, J. M. Kleijn, F. A. M. Leermakers, J. Sprakel, Chem. Commun. 2014, 50, $12197-12200$.

[32] W. Richtering, Langmuir 2012, 28, 17218-17229; M. Faulde, E. Siemes, D. Wöll, A. Jupke, Polymers 2018, 10, 809.

[33] F. A. Plamper, W. Richtering, Acc. Chem. Res. 2017, 50, $131-$ 140.

[34] B. R. Saunders, Langmuir 2004, 20, 3925-3932. 
[35] W. Richtering, B. R. Saunders, Soft Matter 2014, 10, 3695-3702.

[36] A. P. H. Gelissen, A. Oppermann, T. Caumanns, P. Hebbeker, S. K. Turnhoff, R. Tiwari, S. Eisold, U. Simon, Y. Lu, J. Mayer, W. Richtering, A. Walther, D. Wöll, Nano Lett. 2016, 16, 7295 7301.

[37] G. M. Conley, P. Aebischer, S. Nöjd, P. Schurtenberger, F. Scheffold, Sci. Adv. 2017, 3, e1700969.

[38] G. M. Conley, S. Nöjd, M. Braibanti, P. Schurtenberger, F. Scheffold, Colloids Surf. A 2016, 499, 18-23.

[39] S. Bergmann, O. Wrede, T. Huser, T. Hellweg, Phys. Chem. Chem. Phys. 2018, 20, 5074-5083.

[40] M. Irie, T. Fukaminato, K. Matsuda, S. Kobatake, Chem. Rev. 2014, 114, 12174-12277.

[41] J. Zhang, H. Tian, Adv. Opt. Mater. 2018, 6, 1701278.

[42] J. Maier, M. Pärs, T. Weller, M. Thelakkat, J. Köhler, Sci. Rep. 2017, 7, 41739 .

[43] O. Nevskyi, D. Sysoiev, J. Dreier, S. C. Stein, A. Oppermann, F. Lemken, T. Janke, J. Enderlein, I. Testa, T. Huhn, D. Wöll, Small 2018, 14, 1703333 .

[44] Y. Arai, S. Ito, H. Fujita, Y. Yoneda, T. Kaji, S. Takei, R. Kashihara, M. Morimoto, M. Irie, H. Miyasaka, Chem. Commun. 2017, 53, 4066-4069.

[45] O. Nevskyi, D. Sysoiev, A. Oppermann, T. Huhn, D. Wöll, Angew. Chem. Int. Ed. 2016, 55, 12698-12702; Angew. Chem. 2016, $128,12890-12894$.
[46] K. Uno, H. Niikura, M. Morimoto, Y. Ishibashi, H. Miyasaka, M. Irie, J. Am. Chem. Soc. 2011, 133, 13558-13564.

[47] S.-J. Lim, C.-J. Carling, C. C. Warford, D. Hsiao, B. D. Gates, N. R. Branda, Dyes Pigm. 2011, 89, 230-235.

[48] K. I. Mortensen, L. S. Churchman, J. A. Spudich, H. Flyvbjerg, Nat. Methods 2010, 7, 377-381.

[49] R. Kashihara, M. Morimoto, S. Ito, H. Miyasaka, M. Irie, J. Am. Chem. Soc. 2017, 139, 16498-16501.

[50] L. Holtzer, T. Meckel, T. Schmidt, Appl. Phys. Lett. 2007, 90, 053902; B. Huang, W. Q. Wang, M. Bates, X. W. Zhuang, Science 2008, 319, 810-813.

[51] G. T. Dempsey, J. C. Vaughan, K. H. Chen, M. Bates, X. Zhuang, Nat. Methods 2011, 8, 1027-1036.

[52] M. Ovesný, P. Křížek, J. Borkovec, Z. Švindrych, G. M. Hagen, Bioinformatics 2014, 30, 2389-2390.

[53] X. Wu, R. H. Pelton, A. E. Hamielec, D. R. Woods, W. McPhee, Colloid Polym. Sci. 1994, 272, 467-477. 